\title{
Microjet Generator for Highly Viscous Fluids
}

\author{
Hajime Onuki, Yuto Oi, and Yoshiyuki Tagawa* \\ Department of Mechanical Systems Engineering, Tokyo University of Agriculture and Technology, \\ Naka-cho 2-24-16, Koganei, Tokyo 184-8588, Japan
}

(Received 18 October 2017; revised manuscript received 1 January 2018; published 31 January 2018)

This paper describes a simple system for generating a highly viscous microjet. The jet is produced inside a wettable thin tube partially submerged in a liquid. The gas-liquid interface inside the tube, which is initially concave, is kept much deeper than that outside the tube. An impulsive force applied at the bottom of a liquid container leads to significant acceleration of the liquid inside the tube followed by flow focusing due to the concave interface. The jet generation process can be divided into two parts that occur in different time scales, i.e., the impact interval [impact duration $\leq O\left(10^{-4}\right) \mathrm{s}$ ] and the focusing interval [focusing duration $\left.\gg O\left(10^{-4}\right) \mathrm{s}\right]$. During the impact interval, the liquid accelerates suddenly due to the impact. During the focusing interval, the microjet emerges due to flow focusing. In order to explain the sudden acceleration inside the tube during the impact interval, we develop a physical model based on a pressure impulse approach. Numerical simulations confirm the proposed model, indicating that the basic mechanism of the acceleration of the liquid due to the impulsive force is elucidated. Remarkably, the viscous effect is negligible during the impact interval. In contrast, during the focusing interval, the viscosity plays an important role in the microjet generation. We experimentally and numerically investigate the velocity of microjets with various viscosities. We find that higher viscosities lead to reduction of the jet velocity, which can be described by using the Reynolds number (the ratio between the inertia force and the viscous force). This device may be a starting point for next-generation technologies, such as high-viscosity inkjet printers including bioprinters and needle-free injection devices for minimally invasive medical treatments.

DOI: 10.1103/PhysRevApplied.9.014035

\section{INTRODUCTION}

Technologies for generating liquid jets [1] are utilized in important modern devices such as inkjet printers [2-5]. However, most existing printers can eject only lowviscosity liquids of up to $20 \mathrm{~mm}^{2} / \mathrm{s}$, which is about 20 times the viscosity of water. This limitation causes serious problems such as blurring and color dulling. In order to solve these problems, a method for generating jets of highly viscous liquids [6,7] is desired. Furthermore, the generation of viscous microjets can open a new door for nextgeneration technologies such as needle-free injection devices [8-10], printed electronics [11], 3D printers [12], and bioprinters [13], since many highly viscous liquids have various functionalities such as adherence and conductivity [14], which most low-viscosity liquids lack.

Recently, we proposed a simple technique for generating highly viscous liquid jets [15]. A schematic illustration of the proposed microjet generator is shown in Fig. 1(a). We now briefly explain the idea for generating highly viscous liquid jets [15], which relies on three tricks: (1) application of an impulsive force on a liquid by applying an impact at

*tagawayo@cc.tuat.ac.jp the bottom of the liquid-filled container, (2) a thin tube inserted into the liquid, where the liquid level inside the tube is set deeper than that outside the tube, and (3) flow focusing at the gas-liquid interface, which initially has a concave shape. A focused liquid jet forms at the interface (a)

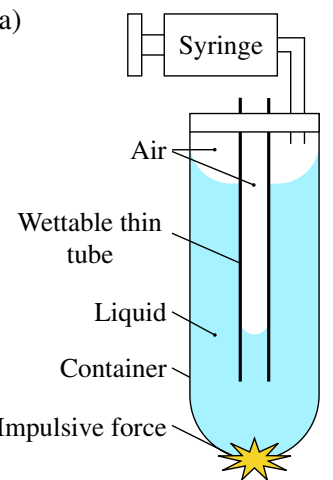

(b)

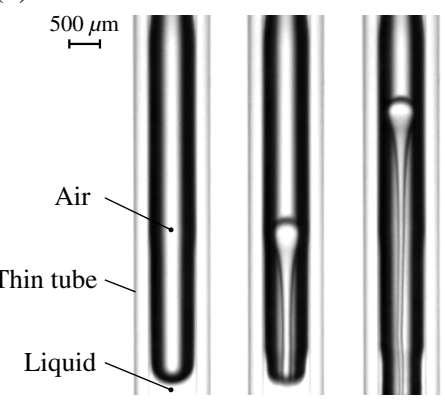

FIG. 1. (a) The highly viscous microjet generator. The liquid level inside the thin tube is kept deeper than that outside the tube by decompressing the air outside the tube. (b) Snapshots of highly viscous microjet generation (kinematic viscosity $\nu=500 \mathrm{~mm}^{2} / \mathrm{s}$ ). The images are acquired every $0.37 \mathrm{~ms}$. 
due to the flow-focusing effect [16-21]. Remarkably, these three tricks enable us to set the velocity of the liquid jet to more than 30 times higher than the initial velocity of the container, resulting in the production of highly viscous liquid jets. The proposed technique has significant advantages with respect to drop-on-demand printers. First, our device can eject a liquid with extremely high viscosity that the present device cannot eject without special treatments for the liquid (e.g., addition of solvent [22], increasing the temperature at the nozzle [4], or conduction of UV [23]). Second, our method consists of a simple structure compared to the ejection methods using a laser [24,25], which leads to the reduction of the cost. However, the size of the liquid jet induced by our method is $O(1) \mathrm{mm}$.

In this study, we develop a device for generating microjets of highly viscous liquids up to $500 \mathrm{~mm}^{2} / \mathrm{s}$ based on the idea described above [see Fig. 1(b)]. In addition, we analyze the generation process of the microjet by dividing the process into two regimes with different time scales, i.e., the impact interval and focusing interval. During the impact interval, the liquid accelerates suddenly due to the impulsive force [impact duration $\leq O\left(10^{-4}\right) \mathrm{s}$ [18]], whereas during the focusing interval, the microjet emerges through flow focusing after the impact interval [focusing duration $\left.\gg O\left(10^{-4}\right) \mathrm{s}\right]$.

\section{BASIC MODEL OF JET GENERATION}

We now explain the physical models for the jet generation during the impact interval and focusing interval.

During the impact interval, we first consider the model for a setup without the thin tube. The incompressible Navier-Stokes equation without external forces except gravity is

$$
\frac{\partial \mathbf{u}}{\partial t}+(\mathbf{u} \cdot \boldsymbol{\nabla}) \mathbf{u}=-\frac{1}{\rho} \boldsymbol{\nabla} p+\nu \nabla^{2} \mathbf{u}+\mathbf{g},
$$

where $\mathbf{u}$ is the velocity of the liquid, $\rho$ is the density, $p$ is the pressure, $\nu$ is the kinematic viscosity, and $\mathbf{g}$ is the acceleration due to gravity. The liquid is accelerated instantly by an impulsive force [26,27] from rest to the velocity $U_{0}$ (referred to as the initial velocity). During impact, the order of the velocity $\mathbf{u}$ is given by the initial velocity $U_{0}$ (approximately $10^{-1} \mathrm{~m} / \mathrm{s}$ ), that of time $t$ is the impact duration $\tau$ (approximately $10^{-4} \mathrm{~s}[18,21]$ ), and that of $\boldsymbol{\nabla}$ is the inverse of the liquid height $z$ (approximately $10^{-2} \mathrm{~m}$ ). We suppose that the kinematic viscosity is much larger than that of water $\left(\nu \sim 10^{-3} \mathrm{~m}^{2} / \mathrm{s}\right)$. Therefore, the orders of each term in the Navier-Stokes equation are $\partial \mathbf{u} / \partial t=O\left(10^{3}\right) \mathrm{m} / \mathrm{s}^{2}, \quad(\mathbf{u} \cdot \boldsymbol{\nabla}) \mathbf{u}=O(1) \mathrm{m} / \mathrm{s}^{2}, \quad \nu \nabla^{2} \mathbf{u}=$ $O(1) \mathrm{m} / \mathrm{s}^{2}$, and $\mathbf{g}=O(10) \mathrm{m} / \mathrm{s}^{2}$. The inertia term $(\mathbf{u} \cdot \boldsymbol{\nabla}) \mathbf{u}$, the viscous term $\nu \nabla^{2} \mathbf{u}$, and the gravity term $\mathbf{g}$ are negligible compared to the other terms. When the device moves only in the vertical direction $z$, temporal integration of Eq. (1) over the impact duration $\tau$ gives

$$
\int_{0}^{\tau} \frac{\partial \mathbf{u}}{\partial t} d t=-\frac{1}{\rho} \int_{0}^{\tau} \nabla p d t=-\frac{1}{\rho} \frac{\partial}{\partial z} \int_{0}^{\tau} p d t
$$

The time integral of pressure on the right-hand side of the equation is called a pressure impulse, which is denoted as

$$
\Pi=\int_{0}^{\tau} p d t
$$

When the liquid is accelerated from rest to the initial velocity $U_{0}$, Eqs. (2) and (3) yield

$$
U_{0}=-\frac{1}{\rho} \frac{\partial \Pi}{\partial z}
$$

As shown in Eq. (4), the initial velocity $U_{0}$ is proportional to the gradient of the pressure impulse in the liquid, which is constant in $z$. Note that the initial velocity $U_{0}$ is not affected by the viscosity $\nu$.

Now we consider the model for a setup with the thin tube in the liquid. Here, we define the parameters shown in Fig. 2: $l_{\text {bottom }}$ is the distance between the bottom of the container and the edge of the thin tube; $l_{\text {middle }}$ is the distance between the edge of the tube and the gas-liquid interface inside the tube; $l_{\text {top }}$ is the distance between the gas-liquid interface inside the tube and that outside the tube. When the cross-sectional area of the thin tube is assumed to be much smaller than that of the container, the motion of the liquid outside the tube is described by Eq. (4). In contrast, the gradient of the pressure impulse inside the tube is significantly larger than that outside the tube since the pressure impulse at the edge of the tube is the same as that outside the tube for a given height (see Fig. 2). The pressure

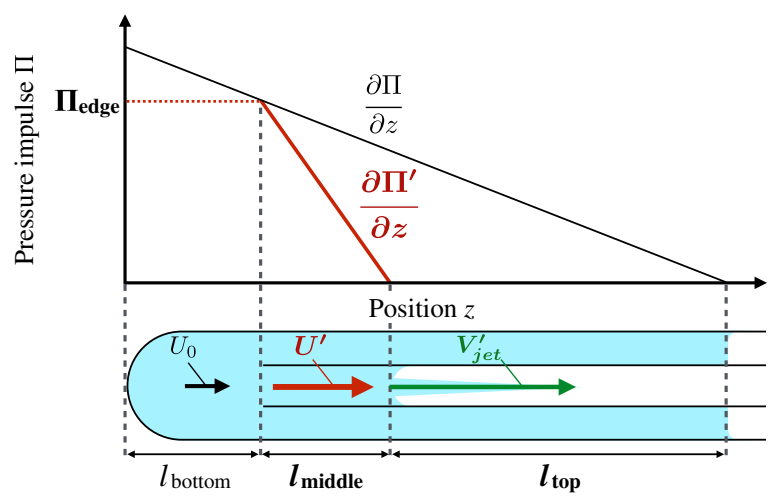

FIG. 2. Physical model based on a pressure impulse approach. The black and red solid lines show the pressure impulse field outside and inside the thin tube, respectively. 
impulse at the edge of the tube $\Pi_{\text {edge }}$ is calculated using a geometrical relation as

$$
\Pi_{\text {edge }}=\left(l_{\text {top }}+l_{\text {middle }}\right) \frac{\partial \Pi}{\partial z} .
$$

Thus, the gradient of the pressure impulse inside the tube $\partial \Pi^{\prime} / \partial z$ is

$$
\frac{\partial \Pi^{\prime}}{\partial z}=\frac{\Pi_{\text {edge }}}{l_{\text {middle }}}=\left(\frac{l_{\text {top }}}{l_{\text {middle }}}+1\right) \frac{\partial \Pi}{\partial z} .
$$

Using Eqs. (4) and (6), the liquid velocity inside the tube $U^{\prime}$ is

$$
U^{\prime}=-\frac{1}{\rho} \frac{\partial \Pi^{\prime}}{\partial z}=\left(\frac{l_{\text {top }}}{l_{\text {middle }}}+1\right) U_{0}
$$

Therefore, during the impact interval, the liquid velocity inside the tube is controlled by tuning $l_{\text {top }}$ and $l_{\text {middle }}$.

During the focusing interval, the liquid jet is produced by the flow-focusing effect [16-21] when the gas-liquid interface reaches velocity $U^{\prime}$. The velocity of the microjet $V_{\text {jet }}^{\prime}$ is proportional to the initial velocity at the interface $U^{\prime}$ [20,21,28]. Using Eq. (7), the jet velocity inside the tube $V_{\text {jet }}^{\prime}$ is

$$
V_{\text {jet }}^{\prime}=\beta U^{\prime}=\beta\left(\frac{l_{\text {top }}}{l_{\text {middle }}}+1\right) U_{0},
$$

where $\beta$ is a constant related to the shape of the interface and viscosity of the liquid. In this paper, $\beta$ is defined as the increment ratio of the jet velocity. The jet velocity $V_{\text {jet }}^{\prime}$ can be varied by changing $l_{\text {top }}$ and $l_{\text {middle. Note that } l_{\text {bottom }} \text { does }}$ not affect the jet velocity $V_{\text {jet }}^{\prime}$.

\section{EXPERIMENTS AND NUMERICAL SIMULATIONS}

\section{A. Experimental setup}

The experimental setup is shown in Fig. 3. A test tube (A-10, Maruemu, 8.0-mm inner diameter) and silicone oil (KF-96 series, Shin-Etsu Chemical; silicone oil, Sigma Aldrich) are used as the container and liquid, respectively. A thin glass tube (FPT-080, Fujiston, 0.5-mm inner diameter, $0.8-\mathrm{mm}$ external diameter) is partially inserted into the liquid in the container. We control the pressure of the sealed air outside the tube using a syringe. The liquid level inside the tube is kept deeper than that outside the tube. To apply the impulsive force, a metal rod is shot (SS400) upward toward the container using a coil gun. After the rod collides with the container, the microjet emerges along the symmetric axis of the interface. The tip velocity of the microjet

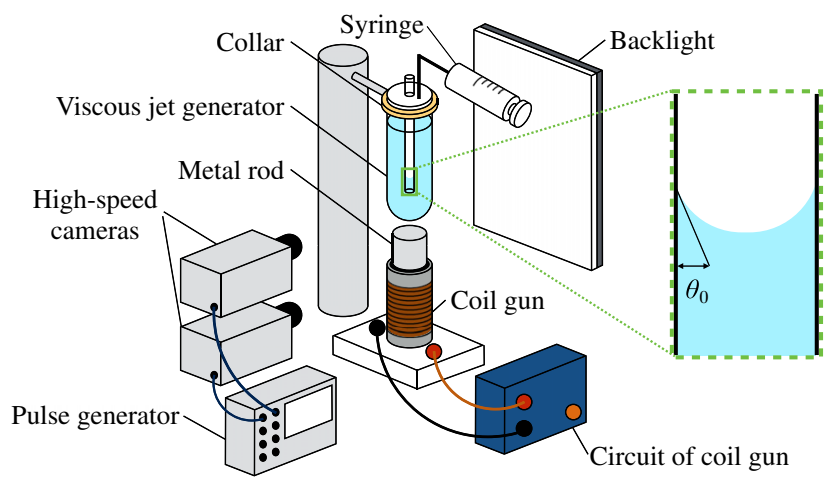

FIG. 3. Sketch of the experimental setup. $\theta_{0}$ is the contact angle between the liquid and the inner wall of the thin tube.

$V_{\text {jet }}^{\prime}$ is calculated to be $0.2 \mathrm{~ms}$ after the jet passes the threephase contact point for the first time, and the initial velocity $U_{0}$ is obtained at $0.8 \mathrm{~ms}$ after the jet passing. We use two high-speed cameras (FASTCAM SA-X, Photron) and a backlight (White Led Backlight, Phlox) to capture the motion of the container and the microjet simultaneously. Both cameras are triggered by a pulse generator (Model 575 Digital Delay and Pulse Generator, BNC). The frame rate of the camera that photographs the motion of the test tube is $12500 \mathrm{fps}$ and that of the camera that focuses on the microjet generation is $50000 \mathrm{fps}$. The experimental parameters are summarized in Table I. We vary $\nu, l_{\text {top }}, l_{\text {middle }}$, and $U_{0}$, and the experiments are conducted five times for each set of conditions. The variation in the microjet velocity $V_{\text {jet }}^{\prime}$ owing to the difference in the contact angle $\theta_{0}$ is estimated to be less than $8 \%$ of $V_{\text {jet }}^{\prime}$.

\section{B. Numerical setup}

We conduct numerical simulations using a commercial software package employing the finite-element method (COMSOL Multiphysics, Keisoku Engineering System Co.). We separately simulate the liquid acceleration during the impact interval and the jet generation during the focusing interval under conditions similar to the experimental ones (see Table I).

The geometry for calculating the liquid acceleration during the impact interval is shown in Fig. 4(a). The inner radius of the thin tube $r$ is $0.25 \mathrm{~mm}$. During the impact interval, the distance between the interface inside the tube and that outside the tube is key to increasing the velocity inside the tube. As an impulsive force, we apply a boundary condition in which the bottom and the side wall accelerate from rest to velocity $U_{0}$ within $1.0 \times 10^{-6} \mathrm{~s}$ [see Fig. 4(b)].

The geometry for reproducing the microjet generation during the focusing interval is shown in Fig. 5(a). During the focusing interval, the duration of the microjet generation is much longer than the acceleration duration during the impact interval. To reduce the computational load, the 
TABLE I. Parameters for the experiments and numerical simulations.

\begin{tabular}{lcccccc}
\hline \hline & $\nu$ & $l_{\text {top }}$ & $l_{\text {middle }}$ & $l_{\text {bottom }}$ & $U_{0}$ & $\theta_{0}$ \\
\hline Experiments & $1-500$ & $39.7-61.7$ & $1.6-10.0$ & 16.0 & $0.3-0.5$ & $28.0-35.4$ \\
Numerical simulations & $1-500$ & $40.0-47.5$ & $2.5-10.0$ & 16.0 & 0.4 & 31.4 \\
\hline \hline
\end{tabular}

simulation for the focusing interval is conducted for the liquid inside the tube. We vary the initial velocity inside the tube $U^{\prime}$ from 2.0 to $20.0 \mathrm{~m} / \mathrm{s}$ and the kinematic viscosity $\nu$ from 1 to $500 \mathrm{~mm}^{2} / \mathrm{s}$. As the sudden acceleration of the liquid, the boundary condition that the pressure changes impulsively within $1.0 \times 10^{-6} \mathrm{~s}$ is enforced at the bottom of the geometry [see Fig. 5(b)]. We adopt the wetted-wall boundary conditions [29] on the inner wall of the thin tube where the three-phase contact line can slide along the wall. In this boundary condition, one considers a virtual wall behind the wall. The distance from the real wall to the virtual wall is called "slip length $l_{s}$," which is set equal to the size of a single mesh in this simulation. The velocity on the virtual wall is zero. This leads to the boundary condition at the real wall as the nonpenetration condition $\mathbf{u} \cdot \mathbf{n}_{\text {wall }}=0$ and the frictional force $\mathbf{F}_{\text {fr }}$ given by

$$
\mathbf{F}_{\mathbf{f r}}=-\frac{\mu}{l_{s}} \mathbf{u}+\sigma\left(\mathbf{n}_{\mathrm{wall}}-\mathbf{n}_{\mathrm{int}} \cos \theta_{0}\right) \delta,
$$

where $\mu$ is the dynamic viscosity, $\sigma$ is the surface tension, $\mathbf{n}_{\text {wall }}$ is the unit vector normal to the wall, $\mathbf{n}_{\text {int }}$ is that normal to interface, and $\delta$ is a function that is nonzero only at the interface $[\delta=6|\phi(1-\phi)||\nabla \phi| ; \phi$ is the level set function]. The $\mathbf{F}_{\text {fr }}$ acts to keep the initial contact angle $\theta_{0}$ at the wall when the three-phase contact line moves with changing the contact angle. This boundary condition about the contact line is sufficient to reproduce the initial production of the microjet due to the small movement of the three-phase contact line. However, it may not be reasonable for the

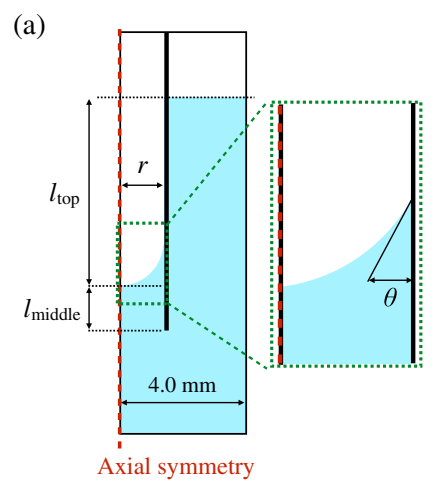

(b)

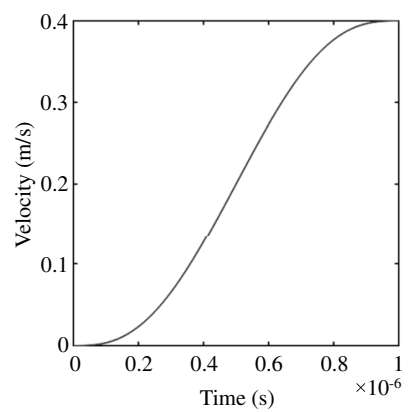

FIG. 4. (a) Geometry for liquid acceleration. (b) The initial impact applied at the bottom of the geometry. The velocity at the bottom and the side wall increases until $U_{0}$ is $1.0 \times 10^{-6} \mathrm{~s}$. reproduction of the elongation of the liquid jet and the formation of the droplet since the three-phase contact line moves significantly for a long period.

The governing equations of the simulation are the incompressible Navier-Stokes equation and equation of continuity. The surface tension is added in the NavierStokes equation term. The level set method $[30,31]$ is used for tracking the gas-liquid interface. Level set methods define the level set function $\phi$, and $\phi=1$ is the liquid phase, $\phi=0$ is the gas phase, and $0<\phi<1$ is the mixed layer of the liquid and the gas. In the mixed layer, the density $\rho$ and the static viscosity $\mu$ are calculated as

$$
\begin{gathered}
\rho=\rho_{l} \phi+\rho_{g}(1-\phi), \\
\mu=\mu_{l} \phi+\mu_{g}(1-\phi) .
\end{gathered}
$$

The subscripts $l$ and $g$ in Eqs. (10) and (11) indicate the liquid and gas phases, respectively. In this paper, we define $\phi=0.5$ to be the gas-liquid interface. Using the level set function, the movement of the interface is expressed as

$$
\frac{\partial \phi}{\partial t}+\mathbf{u} \cdot \boldsymbol{\nabla} \phi=\gamma \boldsymbol{\nabla} \cdot\left(\epsilon \boldsymbol{\nabla} \phi-\phi(1-\phi) \frac{\boldsymbol{\nabla} \phi}{|\boldsymbol{\nabla} \phi|}\right),
$$

where $\gamma$ is the parameter that determines the amount of reinitialization. The $\epsilon$ is the interface thickness defined as half the characteristic mesh size near the interface. In this simulation, we use a triangle mesh, the maximum size of which is $0.05 \mathrm{~mm}$. (a)

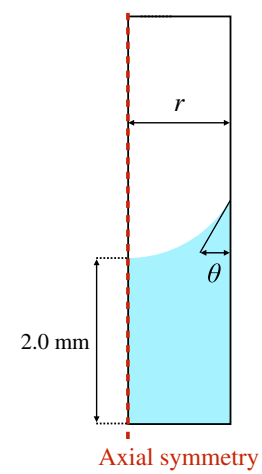

(b)

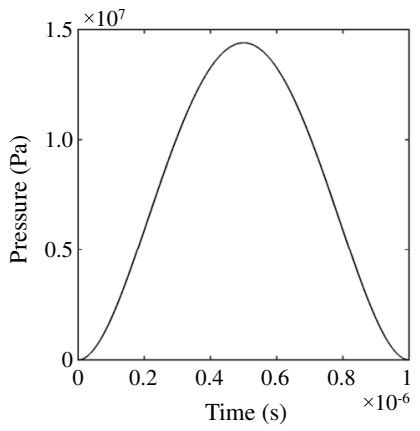

FIG. 5. (a) Geometry for microjet generation. (b) Boundary condition that enforces at the bottom of the geometry as the sudden acceleration of the liquid. 


\section{RESULTS}

\section{A. Impact interval: Acceleration of the liquid}

We show the simulated pressure impulse $\Pi^{*}$ normalized by that at the bottom of the container as a function of the nondimensional vertical position from the bottom $z^{*}$ normalized by that of the interface outside the tube $\left(=l_{\text {bottom }}+l_{\text {middle }}+l_{\text {top }}\right)$ in Fig. 6 as the blue solid line. The gradient of the pressure impulse inside the tube $\partial \Pi^{*} / \partial z^{*}$ is much larger than that outside the tube. The model calculated from Eq. (6) is shown in Fig. 6 as a black solid line. The model is in good agreement with the numerical result. The gradient of the pressure impulse and the velocity inside the tube for each set of conditions are shown in Table II. Here, $U^{\prime *}$ is the velocity inside the tube normalized by that outside the tube $U_{0}$. The velocity inside the tube $U^{*}$ increases up to 17.3 times that outside the tube. The gradient of the pressure impulse $\partial \Pi^{*} / \partial z^{*}$ and the velocity $U^{\prime *}$ are in reasonable agreement with $\left(l_{\text {top }} / l_{\text {middle }}+1\right)$ for all the conditions evaluated, as expected from Eqs. (6) and (7). Remarkably, the gradient of the pressure impulse $\partial \Pi^{\prime *} / \partial z^{*}$ and the velocity $U^{\prime *}$ in each $\left(l_{\text {top }} / l_{\text {middle }}+1\right)$ are almost constant even for the highly viscous liquid. The viscous effect on the sudden acceleration is, thus, negligible during the impact interval.

\section{B. Focusing interval: Microjet generation}

The velocity fields during flow focusing are shown in Fig. 7. Here, the velocity of each point is normalized by the initial velocity applied at the bottom of the geometry $U^{\prime}$. The velocity field just after the impact $(t=1.0 \mu \mathrm{s})$ is almost constant regardless of the viscosity $\nu$; i.e., the viscous effect does not appear just after the impact. This trend agrees with the simulation results shown in Sec. IVA. For a low-viscosity liquid [see Fig. 7(a)], after $t=1 \mu \mathrm{s}$, the

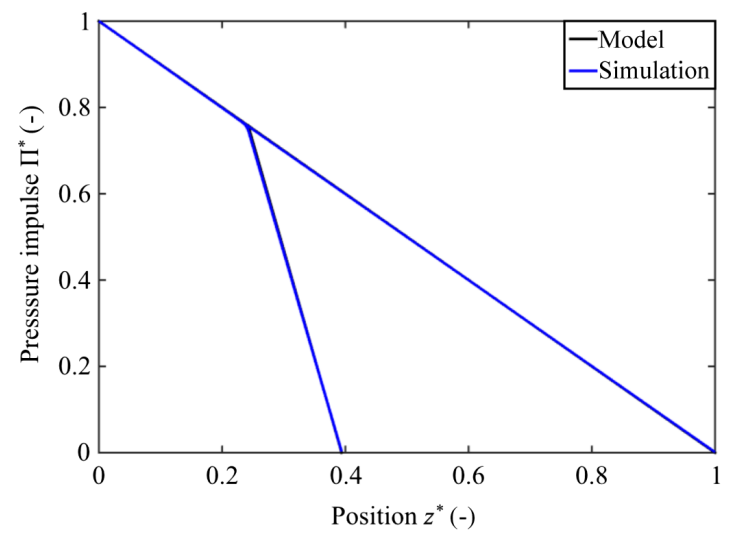

FIG. 6. Relation between the pressure impulse $\Pi^{*}$ and the vertical position from the bottom of the container $z^{*}$ just after impact $\left(t=1.0 \times 10^{-6} \mathrm{~s}\right)$. $l_{\text {top }}$ is $40.0 \mathrm{~mm}, l_{\text {middle }}$ is $10.0 \mathrm{~mm}$, and $l_{\text {bottom }}$ is $16.0 \mathrm{~mm}$. The kinematic viscosity $\nu$ is $1 \mathrm{~mm}^{2} / \mathrm{s}$.
TABLE II. Relation between $\left(l_{\text {top }} / l_{\text {middle }}+1\right)$, the gradient of the pressure impulse, and the velocity inside the tube. $\left|\partial \Pi^{\prime *} / \partial z^{*}\right|$ is the gradient of the pressure impulse inside the tube divided by that outside the tube, and $U^{*}$ is the normalized velocity inside the tube divided by that outside the tube $U_{0}$.

\begin{tabular}{lrrr}
\hline \hline$\left(l_{\text {top }} / l_{\text {middle }}+1\right)(-)$ & $\nu\left(\mathrm{mm}^{2} / \mathrm{s}\right)$ & $\left|\partial \Pi^{* *} / \partial z^{*}\right|(-)$ & $U^{*}(-)$ \\
\hline \multirow{3}{*}{5} & 1 & 4.9 & 4.9 \\
& 100 & 4.9 & 4.9 \\
& 500 & 4.9 & 4.9 \\
10 & 1 & 9.5 & 9.6 \\
& 100 & 9.5 & 9.4 \\
& 500 & 9.5 & 9.4 \\
15 & 1 & 13.8 & 13.4 \\
& 100 & 13.8 & 13.4 \\
& 500 & 13.8 & 13.4 \\
20 & 1 & 18.1 & 17.3 \\
& 100 & 18.0 & 17.3 \\
& 500 & 18.0 & 17.3 \\
\hline \hline
\end{tabular}

velocity of the center of the interface increases up to twice the initial velocity $U^{\prime}$ thanks to the flow-focusing effect. On the other hand, for a high-viscosity liquid [see Fig. 7(b)], the increment of the velocity due to flow focusing does not occur. This result indicates that the liquid viscosity affects the deformation of the interface during flow focusing. Here, we further discuss the viscous effect on microjet velocity.

We show the relation between the microjet velocity $V_{\text {jet }}^{\prime}$ and the initial velocity inside the tube $U^{\prime}$, both of which are obtained from the experiments and the numerical simulations [Fig. 8(a)]. Note that the initial velocity $U^{\prime}$ in the experiments is calculated from Eq. (7). For each viscosity $\nu$, the simulations are in agreement with the experiments, which demonstrates that the simulations can fairly well (a)

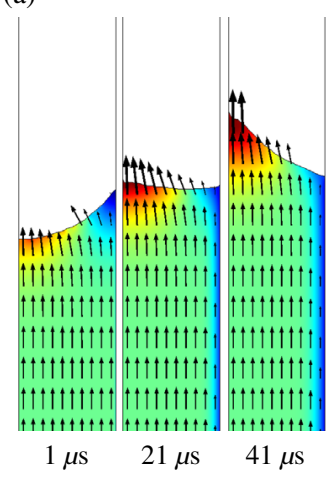

(b)

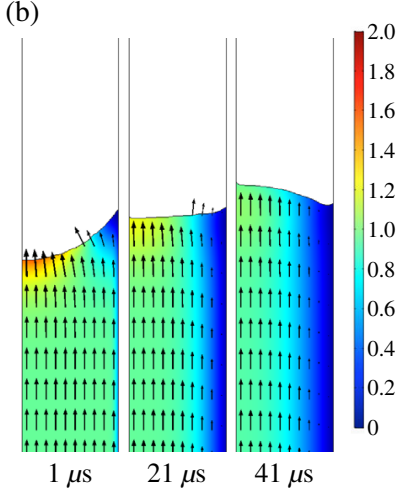

FIG. 7. Vector fields in the liquid during the deformation of the gas-liquid interface. Results with (a) small viscosity $\nu=$ $10 \mathrm{~mm}^{2} / \mathrm{s}$ and (b) large viscosity $\nu=100 \mathrm{~mm}^{2} / \mathrm{s}$. The initial velocity $U^{\prime}=4.0 \mathrm{~m} / \mathrm{s}$ is applied to both conditions. The lefthand image for each condition corresponds to the moment that the sudden acceleration is finished. 
(a)

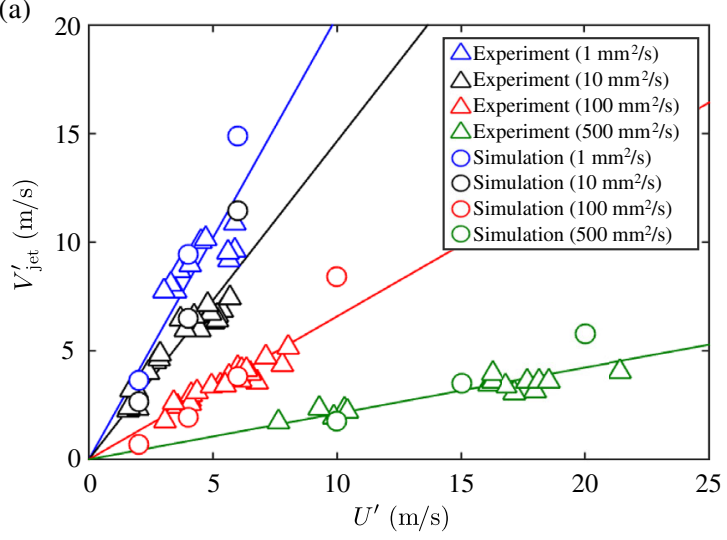

(b)

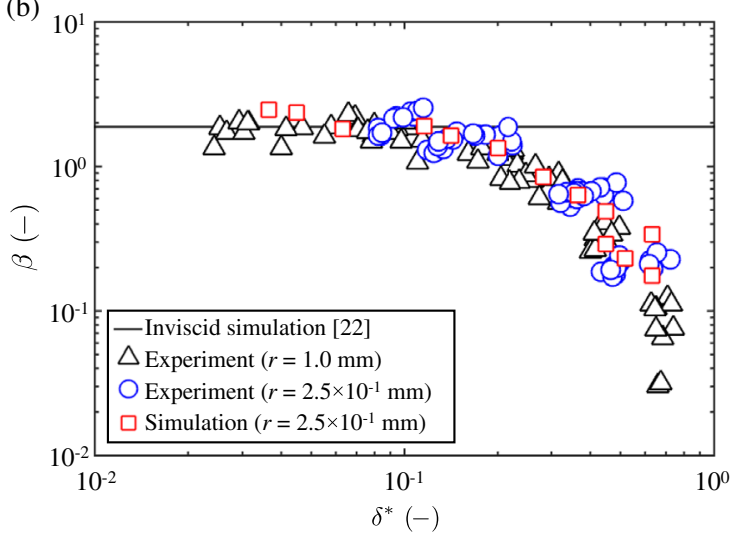

FIG. 8. (a) Relation between the velocity of the microjet $V_{\text {jet }}^{\prime}$ and the initial velocity inside the tube $U^{\prime}$. The triangles are the experimental data, and the circles are the simulations. Blue, black, red, and green correspond to results with $1,10,100$, and $500 \mathrm{~mm}^{2} / \mathrm{s}$, respectively. (b) Increment ratio of the jet velocity $\beta\left(=V_{j}^{\prime} / U^{\prime}\right)$ [see Eq. (8)] vs normalized boundary-layer thickness $\delta^{*}$. The circles and squares are the experimental and simulation results, respectively. The triangles are experimental data obtained using a thin tube with a large inner radius $r=1 \mathrm{~mm}\left(\nu=1-1000 \mathrm{~mm}^{2} / \mathrm{s}\right)$ [15].

reproduce the high-viscosity microjets generated in the experiments.

The model [see Eq. (8)] for each viscosity $\nu$ is shown in Fig. 8(a) as the solid line. Note that $\beta$ in Eq. (8) is fitted to both the experimental and simulation results for each viscosity $\nu$. The increment ratio of the jet velocity $\beta$ decreases with increasing viscosity $\nu$. In other words, during the focusing interval, the viscous effect appears strongly for liquid jets with high viscosity.

To clarify the viscous effect on $\beta$, we consider the development of the boundary layer during the deformation of the interface. It is known that the thickness of the boundary layer $\delta$ is estimated as $\delta=\sqrt{\nu t}$. The focusing time scale $t_{f}$ is estimated as [20]

$$
t_{f} \sim \frac{2 r}{U^{\prime}}
$$

The normalized thickness of the boundary layer $\delta^{*}$ is defined as

$$
\delta^{*}=\frac{\left.\delta\right|_{t=t_{f}}}{r}=\sqrt{\frac{2}{\mathrm{Re}}} .
$$

Here, Reynolds number $\operatorname{Re}$ is calculated as $\operatorname{Re}=U^{\prime} r / \nu$. The normalized thickness of the boundary layer $\delta^{*}$ is inversely proportional to $\sqrt{\mathrm{Re}}$. Because the boundary layer intercepts flow focusing, the increment ratio of the jet velocity $\beta$ will decrease with increasing $\delta^{*}$. The relation between the increment ratio of the jet velocity $\beta$ and the normalized thickness of the boundary layer $\delta^{*}$ is shown in Fig. 8(b). Note that the increment ratio $\beta$ is calculated for each result. The experimental results obtained using a tube with a large inner radius $r=1 \mathrm{~mm}\left(\nu=1-1000 \mathrm{~mm}^{2} / \mathrm{s}\right)$
[15] are also shown in Fig. 8(b). For various values of inner radius $r$ and viscosity $\nu$, the trend of the increment ratio $\beta$ is described by using the normalized thickness of boundary layer $\delta^{*}$, namely, Reynolds number Re. Here, we show the $\beta$ obtained from inviscid simulation [28] in Fig. 8(b) as a black solid line. The inviscid simulation [28] agrees well with our experiments and simulations carried out with small $\delta^{*} \lesssim 10^{-1}(\operatorname{Re} \gtrsim 200)$. On the other hand, for $\delta^{*} \gtrsim 10^{-1}(\operatorname{Re} \lesssim 200)$, the $\beta$ decreases with increasing $\delta^{*}$, which indicates that in this range ( $\operatorname{Re} \lesssim 200$ ), the growth of boundary layer affects the microjet generation during the focusing interval.

\section{CONCLUSION}

We propose a device for generating highly viscous microjets based on some simple tricks: (1) application of an impulse on the bottom of the container, (2) a submerged thin tube, for which the liquid level inside the tube is set deeper than that outside the tube, and (3) preparation of a concave interface for the generation of flow focusing. We conduct the microjet generation experiments and find that our device is able to produce highly viscous liquid microjets (up to $500 \mathrm{~mm}^{2} / \mathrm{s}$ ). To determine the generation mechanism, we divide the process into two parts: the impact interval is the period during which the liquid is suddenly accelerated due to the impulsive force, and the focusing interval is the period during which the liquid emerges as the microjet due to flow focusing. During the impact interval, we focus on the pressure impulse just after sudden acceleration. We develop a physical model and validate it by comparing it with the numerical simulations. We find that the model is able to describe the simulated pressure impulse fields, which indicates that the basic 
mechanism of the device is well understood. During the impact interval, the effect of viscosity is negligible.

In contrast, higher viscosities decrease the velocity of the microjet during the focusing interval. To reveal the mechanism, we consider the development of the boundary layer since the viscosity interrupts flow focusing. Remarkably, the decrement of the jet velocity is found to be described by using Reynolds number Re.

It is worth mentioning that the device is capable of ejecting the jets in multiple directions. This enables us to print viscous liquids to an object with irregular surfaces. Thanks to its simple structure, we can easily produce a hand-sized device at low cost.

\section{ACKNOWLEDGMENTS}

This work is supported by JSPS KAKENHI Grants No. 26709007, No. 17H01246, and No. 17J06711.

\section{APPENDIX A: DROPLET PINCHING}

We experimentally observe the pinch-off process of the droplets. The mechanism of the droplet pinch-off can be described by the end pinching theory [32] or the capillary instability [33]. If the size of droplet $d_{d}$ is given by the end pinching theory [32] in the inviscid limit, the ratio of the drop diameter $d_{d} / r$ is determined by Weber number $\mathrm{We}_{0}\left(=\rho U^{\prime 2} r / \sigma\right)$ as

$$
\frac{d_{d}}{r} \propto \mathrm{We}_{0}^{-1 / 7} \alpha^{2} .
$$

Here, we assume that the strain ratio $\alpha$ is constant. We show the relation between the drop diameter $d_{d}$ and Weber number $\mathrm{We}_{0}$ in Fig. 9(a). The slope of the drop size $d_{d}$ fairly agrees with $\mathrm{We}_{0}^{-1 / 7}$ as predicted by Eq. (A1). Nevertheless, the drop size $d_{d}$ slightly increases with the viscosity $\nu$. To further discuss the pinching mechanism, we examine the pinching time. The pinching time $t_{p}$ satisfies

$$
t_{p} \frac{U^{\prime}}{r} \propto \mathrm{We}_{0}^{2 / 7} \alpha^{-3 / 7}
$$

from end pinching theory [32]. We show the pinching time $t_{p}$ with various Weber numbers $\mathrm{We}_{0}$ in Fig. 9(b). The pinching time $t_{p}$ fairly shows the trend of $\mathrm{We}_{0}^{2 / 7}$ predicted by Eq. (A2) although the coefficient for $1-\mathrm{mm}^{2} / \mathrm{s}$ liquid $(0.53)$ and that for $10 \mathrm{~mm}^{2} / \mathrm{s}(1.18)$ are clearly different. On the other hand, if the pinching mechanism is given by the capillary instability [33], the pinching time $t_{p}$ is estimated as the capillary time scale $t_{c}$ (approximately $\mu r / \sigma$ ) shown in Fig. 9(b). The dotted line of $t_{c}$ significantly differs from the experiments. Therefore, our liquid jet overall follows the end pinching mechanism [32], while the viscous effect is clearly seen.

\section{APPENDIX B: NON-NEWTONIAN JET}

We experimentally produce jets of non-Newtonian liquids (polyacrylamide $1.0 \mathrm{wt} \%$, sodium polyacrylate $0.5 \mathrm{wt} \%$, and xanthan gum $0.3 \mathrm{wt} \%)$. The normalized jet velocity $\left(V_{\text {jet }}^{\prime} / U^{\prime}\right)$ increases with $\left(l_{\text {top }} / l_{\text {middle }}+1\right)$ as predicted by the proposed model [see Fig. 10(b)]. The effect of the non-Newtonian characteristic appears on the increment ratio of jet velocity $\left(V_{\text {jet }}^{\prime} / U^{\prime}\right)$. These results show that our device is applicable to typical non-Newtonian liquids used in real applications.
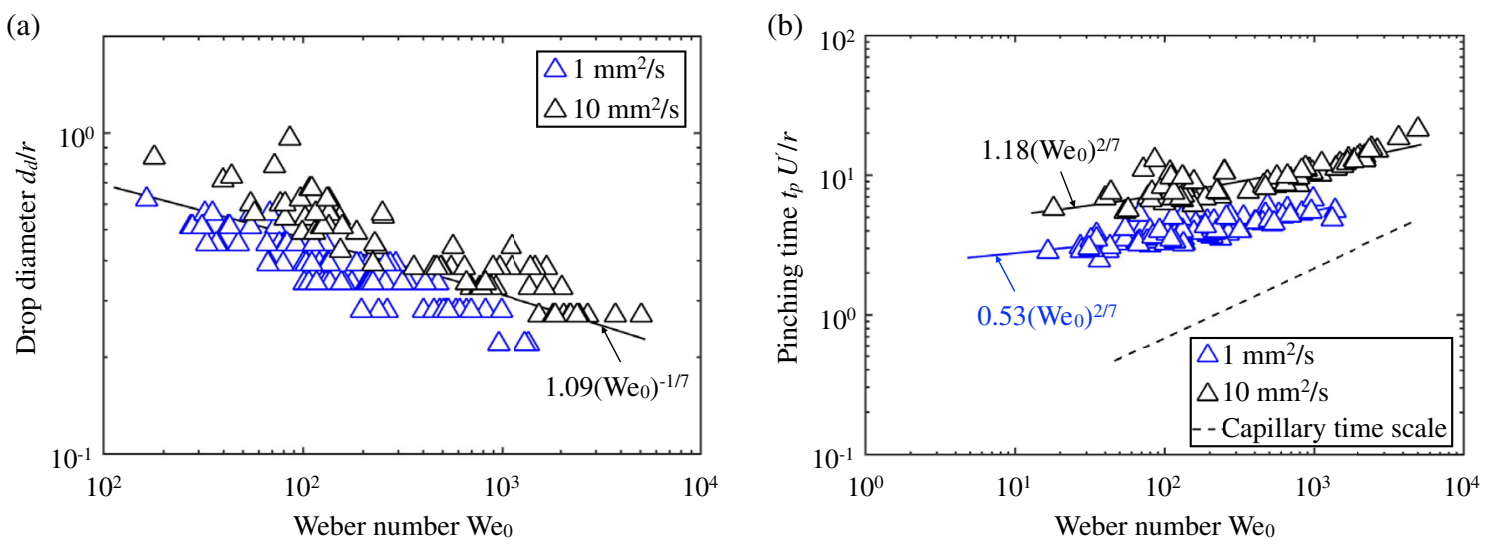

FIG. 9. The results of the drop diameter $d_{d}$ and the pinching time $t_{p}$. (a) The normalized diameter of the droplets $d_{d} / r$ vs Weber number $\mathrm{We}_{0}$. The black solid line is the proportional fitting of the relation between the drop diameter $d_{d}$ and Weber number We $\mathrm{W}_{0}$ [see Eq. (A1)]. (b) The normalized pinching time $t_{p} U^{\prime} / r$ vs Weber number $\mathrm{We}_{0}$. Solid lines are the proportional fitting of the relation between the pinching time $t_{p}$ and Weber number $\mathrm{We}_{0}$ in each viscosity [see Eq. (A2)]. The dotted line shows the capillary time scales $t_{c}$ with the kinematic viscosity $\nu=10 \mathrm{~mm}^{2} / \mathrm{s}$. 
(a)

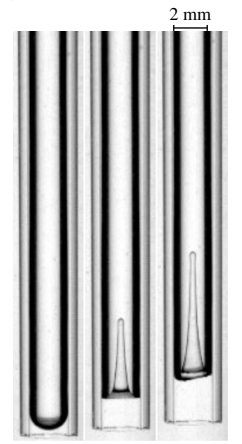

(b)

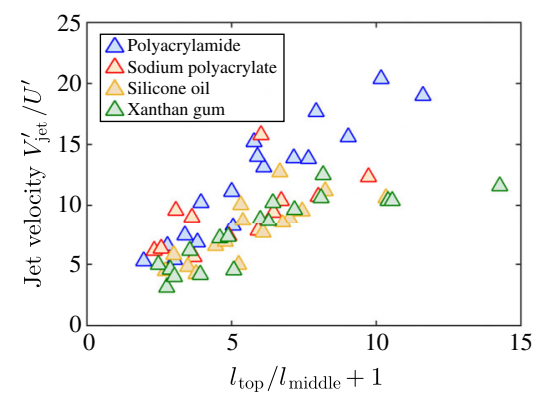

FIG. 10. The jet generation with non-Newtonian liquid. (a) Snapshots of the jet generation with xanthan gum solution $(0.3 \mathrm{wt} \%)$. Each image is taken $1.0 \mathrm{~ms}$ apart. (b) The normalized jet velocity $V_{\text {jet }}^{\prime} / U^{\prime}$ vs $\left(l_{\text {top }} / l_{\text {middle }}+1\right)$ with various nonNewtonian liquids.

[1] J. Eggers and E. Villermaux, Physics of liquid jet, Rep. Prog. Phys. 71, 036601 (2008).

[2] A. van der Bos, M.-J. van der Meulen, T. Driessen, M. van der Berg, H. Reinten, H. Wijshoff, M. Versluis, and D. Lohse, Velocity Profile inside Piezoacoustic Inkjet Droplets in Flight: Comparison between Experiment and Numerical Simulation, Phys. Rev. Applied 1, 014004 (2014).

[3] G. D. Martin, S. D. Hoath, and I. M. Hutchings, Inkjet printing - the physics of manipulating liquid jets and drops, J. Phys. Conf. Ser. 105, 012001 (2008).

[4] H. Wijshoff, The dynamics of the piezo inkjet printhead operation, Phys. Rep. 491, 77 (2010).

[5] O. A. Basaran, H. Gao, and P. P. Bhat, Nonstandard inkjets, Annu. Rev. Fluid Mech. 45, 85 (2013).

[6] H. B. Zhang, S. N. Jayasinghe, and M. J. Edirisinghe, Electrically forced micro threading of highly viscous dielectric liquids, J. Electrost. 64, 355 (2006).

[7] Y. Y. Peng, C. P. Chen, and T. H. Gau, Droplet ejection device for a highly viscous liquid, U.S. Patent No. 7,997,689 (16, August, 2011).

[8] S. Mitragotri, Current status future prospects of needle-free liquid jet injectors, Nat. Rev. Drug Discov. 5, 543 (2006).

[9] Y. Tagawa, N. Oudalov, A. El Ghalbzouri, C. Sun, and D. Lohse, Needle-free injection into skin and soft matter with highly focused microjets, Lab Chip 13, 1357 (2013).

[10] Y. Gong, S. Labh, Y. Jin, H.-Y. Diao, X.-L. Li, Z.-Y. Liu, and Y.-L. Shi, Needle-free injection of 5-aminolevulinic acid in photodynamic therapy for the treatment of nonmelanoma skin cancer, Dermatol. Ther. 29, 255 (2016).

[11] C. W. Visser, R. Pohl, C. Sun, G. W. Römer, B. Huis in't Veld, and D. Lohse, Toward 3D printing of pure metals by laser-induced forward transfer, Adv. Mater. 27, 4087 (2015).

[12] M. Vaezi, H. Seitz, and S. Yang, A review on 3D microadditive manufacturing technologies, International Journal of Advanced Manufacturing Technology 67, 1721 (2013).

[13] S. V. Murphy and A. Atala, 3D bioprinting of tissues and organs, Nat. Biotechnol. 32, 773 (2014).

[14] D. H. Ahmed, H. J. Sung, and D. S. Kim, Simulation of nonNewtonian ink transfer between two separating plates for gravure-offset printing, Int. J. Heat Fluid Flow 32, 298 (2011).

[15] H. Onuki and Y. Tagawa, A highly-viscous liquid jet generator, Trans. J. Multiphase Flow (in Japanese) 29, 335 (2015).

[16] G. Birkhoff, D. P. MacDougall, E. M. Pugh, and S. G. Taylor, Explosives with lined cavities, J. Appl. Phys. 19, 563 (1948).

[17] J.H. Milgram, The motion of a fluid in a cylindrical container with a free surface following vertical impact, J. Fluid Mech. 37, 435 (1969).

[18] A. Antkowiak, N. Bremond, S. Le Dizès, and E. Villermaux, Short-term dynamics of a density interface following an impact, J. Fluid Mech. 577, 241 (2007).

[19] R. Bergmann, E. De Jong, J. B. Choimet, D. van der Meer, and D. Lohse, The origin of the tubular jet, J. Fluid Mech. 600, 19 (2008).

[20] Y. Tagawa, N. Oudalov, C. W. Visser, I. R. Peters, D. van der Meer, C. Sun, A. Prosperetti, and D. Lohse, Highly Focused Supersonic Microjets, Phys. Rev. X 2, 031002 (2012).

[21] A. Kiyama, Y. Noguchi, and Y. Tagawa, The generation of a liquid jet induced by a pressure impulse (experimental study on the jet velocity), Trans. JSME (in Japanese) 80, FE0151 (2014).

[22] M. Singh, H. M. Haverinen, P. Dhagat, and G. E. Jabbour, Inkjet printing-process and its applications, Adv. Mater. 22, 673 (2010).

[23] D. Zhai, T. Zhang, J. Guo, X. Fang, and J. Wei, Water-based ultraviolet curable conductive inkjet ink containing silver nano-colloids for flexible electronics, Colloids Surf. A 424, 1 (2013).

[24] P. Delrot, M. A. Modestino, F. Gallaire, D. Psaltis, and C. Moser, Inkjet Printing of Viscous Monodisperse Microdroplets by Laser-Induced Flow Focusing, Phys. Rev. Applied 6, 024003 (2016).

[25] C. Boutopoulos, I. Kalpyris, E. Serpetzoglou, and I. Zergioti, Laser-induced forward transfer of silver nanoparticle ink: Time-resolved imaging of the jetting dynamics and correlation with the printing quality, Microfluid. Nanofluid. 16, 493 (2014).

[26] G. K. Batchelor, An Introduction to Fluid Dynamics (Cambridge University Press, Cambridge, England, 1967).

[27] M. J. Cooker and D. H. Peregrine, Pressure-impulse theory for liquid impact problems, J. Fluid Mech. 297, 193 (1995).

[28] I. R. Peters, Y. Tagawa, N. Oudalov, C. Sun, A. Prosperetti, D. Lohse, and D. van der Meer, Highly focused supersonic microjets: Numerical simulations, J. Fluid Mech. 719, 587 (2013).

[29] S. Bashir, J. M. Rees, and W. B. Zimmerman, Simulations of microfluidic droplet formation using the two-phase level set method, Chem. Eng. Sci. 66, 4733 (2011).

[30] E. Olsson and G. Kreiss, A conservative level set method for two phase flow, J. Comput. Phys. 210, 225 (2005). 
[31] E. Olsson, G. Kreiss, and S. Zahedi, A conservative level set method for two phase flow II, J. Comput. Phys. 225, 785 (2007).

[32] J. M. Gordillo and S. Gekle, Generation and breakup of Worthington jets after cavity collapse. Part 2. Tip breakup of stretched jets, J. Fluid Mech. 663, 331 (2010).

[33] H. A. Stone and L. G. Leal, Relaxation and breakup of an initially extended drop in an otherwise quiescent fluid, J. Fluid Mech. 198, 399 (1989). 